\title{
Diagnostic Accuracy of Platelet Count and Platelet Indices in Noninvasive Assessment of Fibrosis in Nonalcoholic Fatty Liver Disease Patients
}

\author{
Tamara Milovanovic Alempijevic, ${ }^{1,2}$ Milica Stojkovic Lalosevic, ${ }^{1}$ Igor Dumic, ${ }^{3,4}$ \\ Nevena Jocic, ${ }^{1}$ Aleksandra Pavlovic Markovic, ${ }^{1,2}$ Sanja Dragasevic, ${ }^{1}$ Ivana Jovicic, ${ }^{1}$ \\ Snezana Lukic, ${ }^{1,2}$ Dragan Popovic, ${ }^{1,2}$ and Tomica Milosavljevic ${ }^{1,2}$ \\ ${ }^{1}$ Clinic of Gastroenterology and Hepatology, Clinical Center of Serbia, 11000 Belgrade, Serbia \\ ${ }^{2}$ Faculty of Medicine, University of Belgrade, 11000 Belgrade, Serbia \\ ${ }^{3}$ Mayo Clinic College of Medicine and Sciences, Rochester, MN, USA \\ ${ }^{4}$ Mayo Clinic Health System, Eau Claire, WI, USA
}

Correspondence should be addressed to Tamara Milovanovic Alempijevic; tamara.alempijevic@med.bg.ac.rs

Received 6 September 2017; Accepted 3 December 2017; Published 31 December 2017

Academic Editor: Dan Dumitrascu

Copyright (C) 2017 Tamara Milovanovic Alempijevic et al. This is an open access article distributed under the Creative Commons Attribution License, which permits unrestricted use, distribution, and reproduction in any medium, provided the original work is properly cited.

Objective. Keeping in mind the rising prevalence of nonalcoholic fatty liver disease (NAFLD) and the need to establish noninvasive tests for its detection, the aim of our study was to investigate whether platelet count (PC), mean platelet volume (MPV), and platelet distribution width (PDW) can predict the presence of liver fibrosis in this group of patients. Methods. In 98 patients with NAFLD and 60 healthy volunteers, complete blood counts with automated differential counts were performed and values of PC, PDW, MPV, and PCT were analyzed. Results. Patients with NAFLD had lower PC and higher MPV, PCT, and PDW compared to the controls $(P<0.05)$. When NAFLD group was stratified according to severity of liver fibrosis, there was a statistically significant difference in the average values of PDW and PC between the groups $(P<0.05)$. Conclusion. Patients with NAFLD have significantly higher values of PCT, PDW, and MPV when compared to the healthy controls. Further studies are needed to establish their potential use for prediction of the degree of liver steatosis and fibrosis in NAFLD patients.

\section{Introduction}

Nonalcoholic fatty liver disease (NAFLD) is the leading cause of chronic liver disease worldwide, affecting 20-30\% of population in Western countries [1]. NAFLD is a spectrum from simple steatosis with favorable prognosis to nonalcoholic steatohepatitis (NASH), which may progress to cirrhosis and its complications [2]. The hallmark of NAFLD is intrahepatic deposition of triglycerides and the leading factors in this process are insulin resistance and energy misbalance. NAFLD is considered as hepatic manifestation of the metabolic syndrome [3]. Although a liver biopsy is the gold standard for establishing the diagnosis of NAFLD, we must be aware of its invasiveness, patients' discomfort, risk of severe complications, and its high cost. Bearing in mind the rising prevalence of NAFLD and the fact that it has become one of the most common indications for liver transplantation, there is a need to establish noninvasive diagnostic markers for early detection and monitoring of the disease progression [4-6]. Several noninvasive scores for predicting liver fibrosis have already been described: the aspartate aminotransferase to alanine aminotransferase ratio (AAR), the aspartate aminotransferase to platelet ratio index (APRI), fibrosis index (FI), fibrosis-cirrhosis index (FCI), and FIB-4 index (based on age, aspartate and alanine aminotransferase, and platelet counts). Despite the suggestion that these scores correlate with degree of liver fibrosis, there is no sufficient data supporting their everyday use, yet.

Platelets, along their well-known role in hemostasis, are active participants in the process of liver inflammation. They 
promote leukocyte recruitment through hepatic sinusoids and activate effector cells [7]. Additionally, there are studies suggesting that function and morphology of platelets are altered in patients with diabetes mellitus and metabolic syndrome [8]. MPV, PDW, and PCT (platelet indices) are the indicators of platelet function and activation. It has been suggested that values of platelet indices closely correlate with the presence of insulin resistance and its severity and complications $[9,10]$.

The aim of our cross-sectional case control study was to evaluate whether platelet count and platelet indices can accurately predict severe steatosis and liver fibrosis in patients with NAFLD patients and to compare their diagnostic accuracy with the other noninvasive scores that have been already published and validated.

\section{Patients and Methods}

A total of 98 patients diagnosed with NAFLD and 60 sex- and age-matched healthy volunteers without NAFLD were included in this prospective study from March to September 2016. All patients were diagnosed with NAFLD based on history, physical examination, laboratory testing, and ultrasound imaging. The diagnosis of NAFLD required the exclusion of secondary causes of liver disease and daily alcohol consumption ( $\geq 20 \mathrm{~g}$ for men and $>10 \mathrm{~g}$ for women) [2]. The exclusion criteria were the following: age $<18$ years, presence of any other chronic liver disease (CLD), hepatocellular carcinoma, severe chronic extrahepatic disease, hospital admission due to other chronic illnesses, or presence of human immunodeficiency virus infection. All patients provided a written informed consent prior to inclusion in the study.

Data necessary for the diagnosis of the metabolic syndrome [2] were collected from the patients' records. Physical examination of each patient included body weight, height, waist circumference, calculation of body mass index (BMI), and measurement of blood pressure (BP).

Blood samples were collected after 12 hours of fasting. Analyses included PC, platelet indices, liver function tests (AST, ALT, gamma-glutamyl transferase (GGT), and alkaline phosphatase (ALP)), lipid profile, and fasting blood sugar and insulin levels. Analysis of hematological parameters along with platelets and their indices was performed in whole blood anticoagulated with EDTA within 4 hours after collection, using Coulter ${ }^{\circledR}$ LH 750 Hematology Analyzer (Beckman Coulter, USA). Analysis of biochemical parameters was performed using Olympus AU2700 (Olympus Co. Ltd., Tokyo, Japan). For each patient, we calculated homeostasis model assessment-estimated insulin resistance (HOMA-IR) [11].

Sonographic evaluation (US) of hepatic steatosis was performed using five criteria: parenchymal brightness, liver to kidney contrast, deep beam attenuation, bright vessel walls, and gallbladder wall definition [12]. Grading of diffuse hepatic steatosis was used to evaluate the extent of fatty changes in the liver. Grades I to III were defined as follows:

Grade I: increased hepatic echogenicity with visible periportal and diaphragmatic echogenicity
Grade II: increased hepatic echogenicity with imperceptible periportal echogenicity, without obscuration of diaphragm

Grade III: increased hepatic echogenicity with imperceptible periportal echogenicity and obscuration of the diaphragm [13]

For the assessment of steatosis, we used hepatic steatosis index (HSI) and NAFLD liver fat score (NAFLD-LFS) and we used NAFLD fibrosis score (NFS), APRI, FIB-4 index, and BARD score (which takes into account BMI, AAR, and presence of type II diabetes mellitus) for the assessment of fibrosis. All the above scores were calculated using standard formulas on admission (Tables 1 and 2) [11, 12].

We stratified patients with NAFLD into the two groups based on the US findings. Group 1 included patients with mild and moderate steatosis, while group 2 included patients with severe steatosis and possible fibrosis. In the absence of results from a liver biopsy, we used APRI score as validated "gold noninvasive score" to stratify our patients according to the severity of steatosis and fibrosis $[11,12,14]$.

A statistical analysis was performed using SPSS 22.0 (SPSS Inc., Chicago, IL, USA). Basic descriptive statistics included the means, standard deviations, ranges, and percentages. Normality of the distribution was examined by the Kolmogorov-Smirnov test. The differences were considered as statistically significant if the two-tailed $P$ value was less than 0.05 . The sensitivity and specificity as well as the best cutoff value for the platelet indexes were calculated using ROC curves (AUROC).

This study was approved by the Ethics Committee of our institution in keeping with the principles of the Declaration of Helsinki (2000 revision of Edinburgh).

\section{Results}

Clinical, laboratory, and demographic data of patients were summarized in Table 3.

Gender, age, and mean diastolic blood pressure were similar among the NAFLD patients and the control group $(P>0.05)$. NAFLD patients had significantly higher systolic blood pressure, waist circumference, and BMI compared to the controls $(P<0.01)$. Among the biochemical variables, fasting plasma glucose, insulin levels, and triglycerides were significantly higher and high-density lipoprotein was significantly lower in NAFLD group $(P<0.01)$. NAFLD group also had lower PC and higher MPV, PCT, and PDW $(P<0.05)$.

When we stratified NAFLD patients into the two groups, we found a statistically significant difference in the average values of PDW and PC between the groups $(P=0.04$, $P=0.03$, and $P<0.05)$ with PDW cut-off value of 16.18 for the presence of severe steatosis and possible fibrosis with sensitivity of $88.1 \%$ and specificity of $32.6 \%$ and AUROC of 0.688 (Figure 1). There were no differences between these groups with regard to MPV and PCT $(P>0.05)$.

When these groups were further stratified according to APRI score (more than or equal to 0.7 ), we found a statistically significant difference in values of PC, PDW, and 
TABLE 1: Formulas for calculating noninvasive scores for steatosis.

\begin{tabular}{|c|c|}
\hline $\begin{array}{l}\text { Noninvasive index of } \\
\text { steatosis }\end{array}$ & Formula \\
\hline HSI & $8 * \mathrm{ALT} / \mathrm{AST}+\mathrm{BMI}+2$ if $\mathrm{DM}+2$ if female \\
\hline NAFLD-LFS & $\begin{array}{c}-2.89+1.18 * \text { metabolic syndrome }(\text { yes }=1, \text { no }=0)+0.45 * \text { type } 2 \text { DM }(\text { yes }=1, \text { no }=0) \\
+0.15 \mathrm{Io}+0.04 * \text { AST }-0.94 * \text { AST } / \text { ALT }\end{array}$ \\
\hline \multicolumn{2}{|c|}{ HSI: hepatic steatosis index; NAFLD-LFS: NAFLD liver fat score. } \\
\hline $\begin{array}{l}\text { Noninvasive index } \\
\text { of fibrosis }\end{array}$ & Formula \\
\hline NFS & $\begin{array}{c}1.675+0.037 \times \text { age }(\text { years })+0.094 \times \text { BMI }\left(\mathrm{kg} / \mathrm{m}^{2}\right)+1.13 \times \mathrm{IFG} / \text { diabetes }(\text { yes }=1, \text { no }=0)+0.99 \times \text { AST } / \text { ALT ratio } \\
-0.013 \times \text { platelet }\left(\times 10^{9} / \mathrm{l}\right)-0.66 \times \text { albumin }(\mathrm{g} / \mathrm{dl})\end{array}$ \\
\hline APRI & {$[($ AST/upper normal limit AST $) \times 100] /$ platelets $\left(10^{9} / \mathrm{L}\right)$} \\
\hline FIB-4 & $($ Age $\times$ AST $) /($ platelets $\times$ sqr $($ ALT $))$ \\
\hline BARD & $\mathrm{BMI} \geq 28=1 \mathrm{p}, \mathrm{AST} / \mathrm{ALT}$ ratio $(\mathrm{AAR}) \geq 0.8=2 \mathrm{p}, \mathrm{DM}=1 \mathrm{p}$ \\
\hline
\end{tabular}

NFS: NAFLD fibrosis score; APRI: AST platelet ratio index; FIB-4: fibrosis-4; BARD: BMI, AST/ALT ratio, and diabetes [12, 13].

TABLE 3: Clinical, laboratory, and demographic data of NAFLD patients compared to controls.

\begin{tabular}{lccc}
\hline & NAFLD & Control cases & $P$ value \\
\hline Age & $51.8 \pm 14.6$ & $50.4 \pm 14.1$ & $>0.05$ \\
Gender: male/female & $56 / 42$ & $32 / 28$ & $>0.05$ \\
BMI & $29.3 \pm 4.7$ & $26.2 \pm 3.6$ & $<0.01$ \\
Waist circumference & $109.4 \pm 8.9$ & $108.2 \pm 6.6$ & $<0.05$ \\
Systolic BP & $130.3 \pm 8.9$ & $124 \pm 6.6$ & $<0.01$ \\
Diastolic BP & $82.6 \pm 6.4$ & $81.5 \pm 4.4$ & $>0.05$ \\
Glucose & $6.6 \pm 2.04$ & $5.8 \pm 1.5$ & $<0.01$ \\
Urea & $6.5 \pm 5.9$ & $6.6 \pm 7.5$ & $>0.05$ \\
Creatinine & $77.5 \pm 15.2$ & $73.3 \pm 21.1$ & $>0.05$ \\
Total cholesterol & $5.78 \pm 1.1$ & $5.72 \pm 1.4$ & $>0.05$ \\
LDL cholesterol & $3.6 \pm 0.9$ & $2.6 \pm 1.1$ & $<0.01$ \\
HDL cholesterol & $1.2 \pm 0.6$ & $1.7 \pm 0.4$ & $<0.01$ \\
Triglyceride & $2.4 \pm 1.7$ & $1.7 \pm 0.9$ & $<0.01$ \\
AST & $33.1 \pm 19.3$ & $22.3 \pm 11.2$ & $<0.01$ \\
ALT & $46.1 \pm 25.8$ & $22.1 \pm 7.8$ & $<0.01$ \\
ALP & $72.3 \pm 23.0$ & $73 \pm 19.2$ & $>0.05$ \\
GGT & $73.6 \pm 82.9$ & $44 \pm 12.1$ & $<0.01$ \\
WBC & $7.02 \pm 1.7$ & $7.2 \pm 2.6$ & $>0.05$ \\
PC & $218.4 \pm 56.8$ & $255.3 \pm 77.9$ & $<0.01$ \\
MPV & $9.1 \pm 1.3$ & $7.6 \pm 1.1$ & $<0.01$ \\
PDW & $16.7 \pm 0.7$ & $15.9 \pm 0.7$ & $<0.01$ \\
PCT & $0.2 \pm 0.1$ & $0.1 \pm 0.0$ & $<0.01$ \\
\hline
\end{tabular}

PCT between the two groups $(P=0.00, P=0.00, P=0.006$, and $P<0.05$ ) (Table 4).

We found a statistically significant negative correlation between PC and APRI $(P=0.00 ; r=-0.530)$, FIB-4 $(P=$ $0.00 ; r=-0.480)$, and NFS $(P=0.00 ; r=-0.320)$ scores, respectively. Additionally, we found statistically significant negative correlations between PDW and APRI $(P=0.00$;

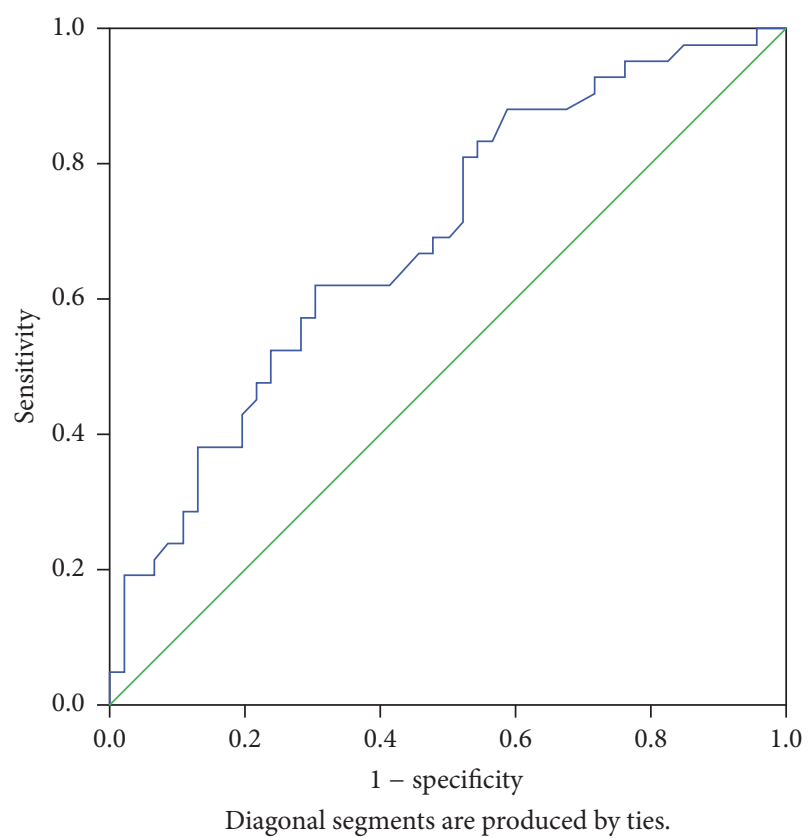

FIgure 1: ROC curve 1. Receiver Operating Characteristic (ROC) Curve illustrates sensitivity and specificity of mean PDW values for detection of mild, moderate, and severe steatosis.

$r=-0.5629)$, FIB-4 $(P=0.00 ; r=-0.553)$, and NFS $(P=0.00 ; r=-0.346)$ scores, respectively.

The results of our study suggest that there is a statistically significant negative correlation between PCT and PDW $(P=$ $0.06 ; r=-0.252)$, as well as a significant positive correlation between PDW and MPV $(P=0.04 ; r=0.261)$.

\section{Discussion}

The ability to determine the degree of the liver steatosis and fibrosis as well as to predict the progression of disease is 
TABLE 4: APRI score according to platelet count and indices.

\begin{tabular}{lccc}
\hline & APRI $\geq 0.7$ & APRI $<0.7$ & $P$ \\
\hline PC (mean \pm SD) & $154.71 \pm 44.92$ & $226.91 \pm 52.90$ & $<0.01$ \\
MVP (mean \pm SD) & $9.21 \pm 1.05$ & $9.04 \pm 1.46$ & 0.581 \\
PCT (mean \pm SD) & $0.14 \pm 0.03$ & $0.22 \pm 0.16$ & $<0.01$ \\
PDW (mean \pm SD) & $16.91 \pm 0.35$ & $16.55 \pm 0.77$ & $<0.01$ \\
\hline
\end{tabular}

essential in the management of patients with NAFLD. A liver biopsy has been used as a gold standard for this purpose over many years. However, its invasive nature, high cost, and risk for development of severe complications (bleeding in particular) resulted in the development of noninvasive tests. These tests consist of different scores derived from various combinations of serologic markers as well as noninvasive imaging modalities [15]. Over the last decade, particularly promising imaging modality has emerged, ultrasound-based transient elastography (TE) with controlled attenuation parameter (CAP). This technique is based on mechanical excitation of the hepatic tissue with monitoring of the resulting tissue response. The fact that fibrotic tissue differs from healthy tissue in the way that it responds to excitation is used to determine level of steatosis and fibrosis. This technique is particularly useful, since it eliminates sampling errors, which was common with liver biopsy, in addition to its noninvasiveness $[16,17]$. However, while it is being used more commonly, high cost of the machine is still an obstacle for use in many developing countries. Hence, there is an ongoing interest for discovery of the cheaper and easily available serologic tests for detection of liver steatosis and fibrosis and estimation of the severity grades. Multiple serologic markers reflective of liver function (AST, ALT, bilirubin, PC, GGT, ALP, and haptoglobin) have been used in combination to formulate diagnostic and prognostic scores that would be an alternative to liver biopsy. While some of them are more simple (AAR), some of them include complicated algorithms and multiple variables (FibroTest).

The current research studies suggest that platelets have a role in the process of liver fibrosis by decreasing expression of the principal fibrogenic cytokine TGF- $\beta$ and by increasing expression of matrix metalloproteinases [18, 19]. Subsequently, an inverse correlation occurs between progression of liver fibrosis and platelets. Taking this into account, PC is presently included in many prognostic scores for fibrosis and cirrhosis of the liver. Some previous studies have described that lower PC is related to the more advanced fibrosis; however, only few of these studies assessed PC in NAFLD patients [20]. Unlike platelets, the platelet indices are not widely investigated as the markers of liver steatosis and fibrosis. They might prove to be very useful in the future as a part of diagnostic scores for detection of liver steatosis and fibrosis in patients with NAFLD.

Our study aimed to determine the association between PC and platelet indices with the presence of fibrosis in NAFLD patients and we found an inverse correlation between PC and liver fibrosis, similar to previously published data $[18,19]$.
Platelet functions can be affected by platelet size, density, other comorbidities, and age. Larger platelets have higher quantity of granules and adhesion receptors, which results in an increase in platelet activation [21]. PDW directly refers to platelet size, changes with platelet activation, and reflects the heterogeneity in platelet morphology [7]. Our study suggests that NAFLD patients have higher values of PDW compared to controls. Study of Cao et al. showed that PC and PDW negatively correlate with the stage of fibrosis, which is in accordance with results that we found in the present study [22].

The results of Ozhan et al. suggest that lower PC and higher MPV are independent predictors of NAFLD [23]. Several independent studies have reported that steatosis was associated with an elevation in MPV [23-27]. A large Korean study has demonstrated a significant association between the presence of NAFLD and higher MPV values in 628 obese volunteers [24]. In our study, the NAFLD group had significantly higher values of MPV compared to the controls, which is similar to the published data.

To the best of our knowledge, there are no studies that investigated potential use of PCT for estimation of the degree of liver steatosis/fibrosis in patients with NAFLD. In the current study, we have not found any significant difference in the values of PCT between NAFLD groups 1 and 2; however, we have found significant difference in the values of PCT between NAFLD patients and the controls. This can be potentially useful as quick and simple parameter for orientation towards patients with suspected NAFLD.

\section{Conclusion}

In conclusion, our study demonstrates that patients with NAFLD have significant increase in the values of PCT, PDW, and MPV. We will need larger studies to investigate potential use of PC and platelet indices and their inclusion in the diagnostic algorithms for noninvasive assessment of degree of steatosis and fibrosis in NAFLD patients. Their use may be beneficial considering that they are simple, easy to measure, and cost-effective and are routinely checked in everyday practice.

\section{Additional Points}

Limitations of the Study. NAFLD diagnosis was not confirmed by liver biopsy and baseline analyses may not reflect patient's condition over prolonged period of time. Additionally, low AUROC suggest that there is a need for a larger cohort.

\section{Conflicts of Interest}

The authors have no conflicts of interest to declare.

\section{References}

[1] S. A. Madan, F. John, and C. S. Pitchumoni, "Nonalcoholic Fatty Liver Disease and Mean Platelet Volume: A Systemic Review and Meta-analysis," Journal of Clinical Gastroenterology, vol. 50, no. 1, pp. 69-74, 2016. 
[2] M. Cengiz, B. A. Candir, G. Yilmaz, G. Akyol, and S. Ozenirler, "Is increased red cell distribution width an indicating marker of nonalcoholic steatohepatitis and fibrotic stage?" World Journal of Gastroenterology, vol. 19, no. 42, pp. 7412-7418, 2013.

[3] European Association for the Study of the Liver (EASL), "EASL-EASD-EASO Clinical Practice Guidelines for the management of non-alcoholic fatty liver disease," Diabetologia, vol. 59, no. 6, pp. 1121-1140, 2016.

[4] D. R. LaBrecque, Z. Abbas, F. Anania et al., "World gastroenterology organisation global guidelines: nonalcoholic fatty liver disease and nonalcoholic steatohepatitis," Journal of Clinical Gastroenterology, vol. 48, no. 6, pp. 467-473, 2014.

[5] S. Bellentani, F. Scaglioni, M. Marino, and G. Bedogni, "Epidemiology of non-alcoholic fatty liver disease," Digestive Diseases, vol. 28, no. 1, pp. 155-161, 2010.

[6] S. Milić, I. Mikolasevic, and I. Krznaric-Zrnic, "Nonalcoholic steatohepatitis: emerging targeted therapies to optimize treatment options," Drug Design, Development and Therapy, vol. 9, pp. 4835-4845, 2015.

[7] A. Chauhan, D. H. Adams, S. P. Watson, and P. F. Lalor, "Platelets: No longer bystanders in liver disease," Hepatology, vol. 64, no. 5, pp. 1774-1784, 2016.

[8] A. Koneti Rao, R. E. Goldberg, and P. N. Walsh, "Platelet coagulant activities in diabetes mellitus. Evidence for relationship between platelet coagulant hyperactivity and platelet volume," Journal of Laboratory and Clinical Medicine, vol. 103, no. 1, pp. 82-92, 1984.

[9] Y. U. Budak, M. Polat, and K. Huysal, "The use of platelet indices, plateletcrit, mean platelet volume and platelet distribution width in emergency non-traumatic abdominal surgery: a systematic review," Biochemia Medica, vol. 26, no. 2, pp. 178-193, 2016.

[10] A. T. Eminler, M. I. Uslan, T. Ayyildiz et al., "Mean platelet volume is an important predictor of hepatitis $\mathrm{C}$ but not hepatitis B liver damage," Journal of Research in Medical Sciences, vol. 20, no. 9, pp. 865-870, 2015.

[11] T. Alempijevic, A. Sokic-Milutinovic, A. P. Markovic et al., "Assessment of metabolic syndrome in patients with primary biliary cirrhosis," Wiener Klinische Wochenschrift, vol. 124, no. 7-8, pp. 251-255, 2012.

[12] S. Kahl, K. Straßburger, B. Nowotny et al., "Comparison of liver fat indices for the diagnosis of hepatic steatosis and insulin resistance," PLoS ONE, vol. 9, no. 4, Article ID A1453, 2014.

[13] C. Das, M. Baruah, and D. Singh, "Imaging of non alcoholic fatty liver disease: A road less travelled," Indian Journal of Endocrinology and Metabolism, vol. 17, no. 6, p. 990, 2013.

[14] A. Unalp-Arida and C. E. Ruhl, "Noninvasive fatty liver markers predict liver disease mortality in the U.S. population," Hepatology, vol. 63, no. 4, pp. 1170-1183, 2016.

[15] A. Wieckowska and A. E. Feldstein, "Diagnosis of nonalcoholic fatty liver disease: Invasive versus noninvasive," Seminars in Liver Disease, vol. 28, no. 4, pp. 386-395, 2008.

[16] J. Foucher, E. Chanteloup, J. Vergniol et al., "Diagnosis of cirrhosis by transient elastography (FibroScan): a prospective study," Gut, vol. 55, no. 3, pp. 403-408, 2006.

[17] J. Ophir, I. Céspedes, H. Ponnekanti, Y. Yazdi, and X. Li, "Elastography: a quantitative method for imaging the elasticity of biological tissues," Ultrasonic Imaging, vol. 13, no. 2, pp. 111134, 1991.

[18] C. Fierbinteanu-Braticevici, I. Dina, A. Petrisor, L. Tribus, L. Negreanu, and C. Carstoiu, "Noninvasive investigations for non alcoholic fatty liver disease and liver fibrosis," World Journal of Gastroenterology, vol. 16, no. 38, pp. 4784-4791, 2010.

[19] A. Gülali, A. Aytekin, K. Buket et al., "Mean Platelet Volume and Red Cell distribution width in Hepatosteatosis," National Journal of Medical Research, vol. 3, no. 3, pp. 264-266, 2013.

[20] Z. Saremi, M. Rastgoo, M. Mohammadifard, B. Bijari, and E. Akbari, "Comparison of platelet number and function between nonalcoholic fatty liver disease and normal individuals," Journal of Research in Medical Sciences, vol. 22, no. 1, p. 75, 2017.

[21] C. H. Kim, S. J. Kim, M. J. Lee et al., "An increase in mean platelet volume from baseline is associated with mortality in patients with severe sepsis or septic shock," PLoS ONE, vol. 10, no. 3, Article ID e0119437, 2015.

[22] W. Cao, C. Zhao, C. Shen, and Y. Wang, "Cytokeratin 18, alanine aminotransferase, platelets and triglycerides predict the presence of nonalcoholic steatohepatitis," PLOS ONE, vol. 8, no. 12, Article ID e82092, 2013.

[23] H. Ozhan, M. Aydin, M. Yazici et al., "Mean platelet volume in patients with non-alcoholic fatty liver disease," Platelets, vol. 21, no. 1, pp. 29-32, 2010.

[24] W.-Y. Shin, D.-H. Jung, J.-Y. Shim, and H.-R. Lee, “The association between non-alcoholic hepatic steatosis and mean platelet volume in an obese Korean population," Platelets, vol. 22, no. 6 , pp. 442-446, 2011.

[25] K. Fang, Y. Cheng, C. Su et al., "Higher platelet counts are associated with metabolic syndrome independent of fatty liver diagnosis," Journal of the Chinese Medical Association, vol. 80, no. 3, pp. 125-132, 2017.

[26] S. Arundhathi, S. Sunitha, and R. Gandham, "Mean Platelet Volume in Non-Alcoholic Fatty Liver Disease in Type 2 Diabetes Mellitus - A Marker of Cardiovascular Risk," International Journal of Biomedical and Advance Research, vol. 7, no. 2, pp. 7678, 2016.

[27] M. Aydin, M. Yazici, O. Yazgan et al., "Mean platelet volume in patients with non-alcoholic fatty liver disease," Platelets, vol. 21, pp. 29-32, 2010. 


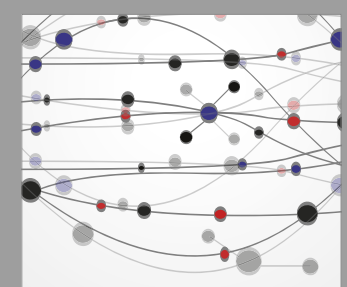

The Scientific World Journal
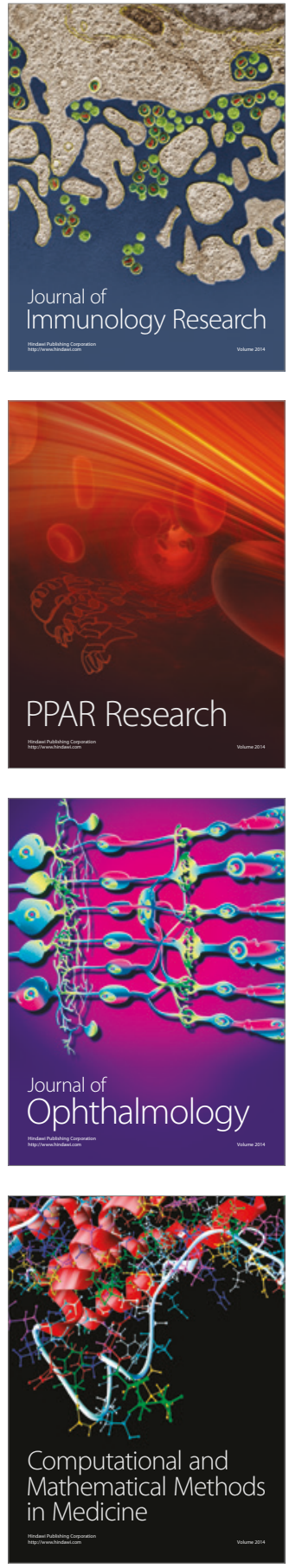

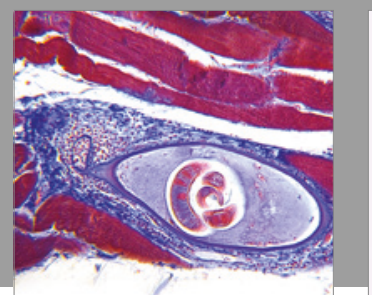

Gastroenterology Research and Practice
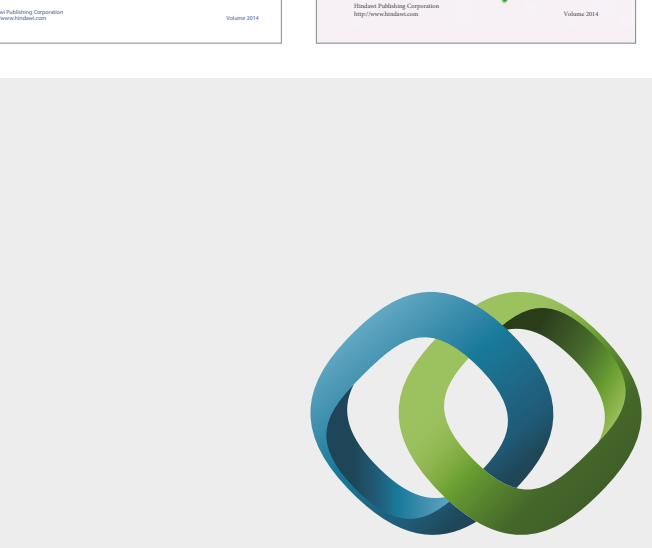

\section{Hindawi}

Submit your manuscripts at

https://www.hindawi.com
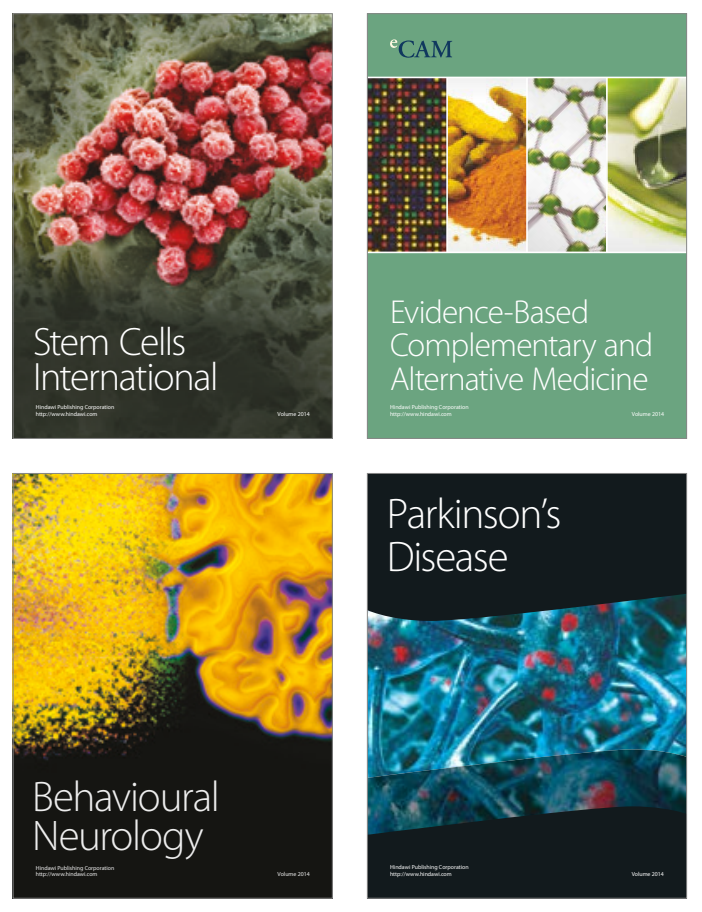
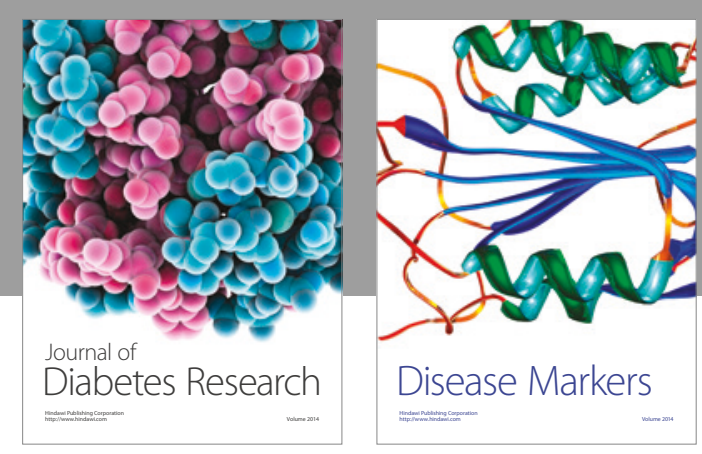

Disease Markers
